\title{
EDITORIAL
}

\section{Complications of transcatheter closure of atrial septal defects}

M S Spence, S A Qureshi

Transcatheter device closure of atrial septal defects has proved to be remarkably successful, and is usually preferred by both patients and parents. But how does it compare with the gold standard of surgical closure?
See end of article for authors' affiliations

Correspondence to: Dr Shakeel A Qureshi, Department of Congenital Heart Disease, Guy's \& St Thomas' Hospital Foundation Trust, St Thomas' Street, London SE1 9RT, UK; Shakeel. Qureshi@gstt.nhs.uk

Published Online First 23 August 2005
$S^{2}$ ecundum type atrial septal defects (ASDs) are the fourth most common congenital heart defect, with an incidence of 3.78 per 10000 live births ${ }^{1}$ and a diagnosed prevalence rate of around $6 \%$ in children. ${ }^{2}$ Although surgical closure of these defects has been the traditional method of treatment, as long ago as 1976, King et al first described successful transcatheter device closure. ${ }^{3}$ Subsequently, there was slow progress in developing a reliable method of device closure. It was not until the 1990s that the technique became a widely accepted and practiced procedure, such that it has now largely replaced surgical closure of secundum ASDs in most centres. This rapid growth in transcatheter device closure has been accompanied by publications from many centres reporting outcome data for this procedure, including its complications. Cognisance of these complications, as longer term follow up data emerges, is important in informing best practice. Although transcatheter closure of ASDs has been remarkably successful, the benchmark against which it should be measured is the traditional surgical method, given its proven efficacy compared with medical management. ${ }^{45}$

\section{COMPLICATIONS AFTER SURGICAL CLOSURE OF SECUNDUM ATRIAL SEPTAL DEFECTS}

There have been no large, truly randomised comparisons of surgery with transcatheter closure of ASDs as the design of such a study is problematic partly because, given a choice between surgery and device closure, parents and patients often prefer the latter method. Nevertheless, surgery is the gold standard against which transcatheter closure of ASDs has been and should be judged, not least because longer term follow up is available for surgical treatment.

A Dutch group have reported longitudinal follow up of 21-33 years (mean 27 years) after surgical closure of secundum ASDs in 135 patients. ${ }^{6}$ There was no cardiovascular mortality, stroke, heart failure, or pulmonary hypertension. However, symptomatic supraventricular tachyarrhythmias occurred in $6 \%$ of patients after 15 years and 5\% needed pacemaker implantation. Thus arrhythmias appear to be the main long term complication after surgical closure of ASDs.
Heart 2005;91:1512-1514. doi: 10.1136/hrt.2004.057562

Early complications after surgery include wound infection, pericardial effusions causing tamponade, and the occasional need for repeat surgery. The median hospital stay is short (four days). A review published in 2003 of 100 consecutive children undergoing surgical closure reported no mortality, but the incidence of postpericardiotomy syndrome was 3\% and of pericardial effusions was $26 \% .^{7}$ Another review published in 2004 of 176 consecutive patients (47 adult and 129 children) undergoing surgical closure reported no mortality. ${ }^{8}$ However, serious perioperative complications included atrial fibrillation (in $10 \%$ of adults and in $1.2 \%$ of children) and post-pericardiotomy syndrome (in $2 \%$ of adults and in $4.7 \%$ of children).

These recent surgical series show that surgical closure is still associated with significant morbidity and complications and therefore is not without risk.

\section{COMPLICATIONS AFTER TRANSCATHETER DEVICE CLOSURE OF SECUNDUM ATRIAL SEPTAL DEFECTS}

Since the launch of the transcatheter interventional era for the treatment of congenital heart defects, there have been many different devices available for transcatheter closure of secundum ASDs, some of which have been modifications of earlier models.

The efficacy of transcatheter closure of these defects has not been as comprehensively demonstrated as for closure of patent arterial ducts. However, the close scrutiny of the results and complications of transcatheter closure of patent arterial ducts is now occurring for ASDs and considerable literature has now been published examining the efficacy of the various closure devices.

\section{Procedure related complications}

Most centres around the world perform this procedure under general anaesthesia in children. This then adds the risks of general anaesthesia to the procedure. For adult patients, the practice with regard to general anaesthesia or sedation is more mixed, particularly with the advent of intracardiac echocardiography which obviates the need for transoesophageal echocardiography, although the cost of intracardiac echocardiography probes has limited its use.

Transcatheter closure is associated with all the general risks inherent in any interventional cardiac catheterisation procedure such as the risk of contrast reactions, vessel or cardiac perforation, and the introduction of infection. There are some complications of the procedure 
which are difficult to quantify and for which data may not be available. Complications of femoral vein access include haematomas that may rarely require blood transfusions and, even more rarely, surgical repair when retroperitoneal haematomas have developed. In practice, these risks may be slightly increased with larger venous sheaths needed for the larger devices and probably in elderly or obese patients. Perclose suture mediated vascular closure devices (Abbott, Abbott Park, Illinois, USA) have been used in selected adult patients requiring larger sheaths. ${ }^{9}$

Chessa et al have reported on a large series of 417 patients who had catheter closure of secundum ASDs of whom 159 received CardioSEAL/STARFlex and 258 Amplatzer septal occluder devices. ${ }^{10}$ Overall, there were $36 / 417$ (8.65\%) complications, of which 11 were major and 25 minor. The most common complication was device embolisation/malposition occurring in $3.5 \%$ of cases. Of the 15 patients in whom devices embolised or were malpositioned, 10 required surgical retrieval while in the remainder the devices were retrieved by catheter techniques. The next most common complication was arrhythmia, which occurred in 11/417 $(2.6 \%)$ of cases. In six of these 11 , atrial fibrillation required electrical cardioversion. Pericardial effusion occurred in two patients, in one case due to cardiac perforation, and for no obvious reason in another. One patient developed thrombus on the left atrial disc of an Amplatzer septal occluder device immediately after deployment, despite full heparinisation. There was one episode of right iliac vein dissection requiring stent implantation and one groin haematoma requiring surgical revision.

There have been many other reports of procedure related complications. These include a report on 236 patients considered for closure with the Amplatzer septal occluder. ${ }^{11}$ Of these, 200 had a successful transcatheter closure and two had a serious procedural related complication (one retroperitoneal haematoma requiring surgery and one air embolism to the right heart after device implantation with no apparent sequelae). In a multicentre UK experience, Chan et al reported on 101 Amplatzer septal occluder procedures performed in 100 patients. ${ }^{12}$ Complications included device embolisation in one patient, transient ST elevation in one, transient atrioventricular block in one, presumed deep vein thrombosis in one, and presumed transient ischaemic attack in one patient. A recent observational study by Krumsdorf et al on a large series of patients suggests that although the overall rate of thrombus formation on closure devices is low, there may be differences in the risk with different devices. ${ }^{13}$ There have also been isolated reports of systemic allergic reaction to nickel-containing atrial septal closure devices.

As with any new technique in clinical use, there is undoubtedly a learning curve related to transcatheter closure of ASDs. Avoidance of complications is dependent on careful case selection, sizing of the defect, selection of the correct size of device, aseptic technique, periprocedural heparinisation, avoidance of air embolism and testing of device stability during the procedure, meticulous attention to antiplatelet treatment, and antibiotic prophylaxis. Vigilance is also required to detect complications early so that remedial action can be taken promptly to minimise the risk of serious adverse outcomes.

\section{Intermediate and longer term complications after transcatheter closure}

A much more worrying complication of cardiac erosion by the Amplatzer septal occluder after catheter closure of ASDs has recently been reported by Amin et al. ${ }^{14}$ Erosions have been identified by the late development of pericardial effusion or even tamponade. Data were collected from 28 cases worldwide and all the erosions occurred near the aortic root. The paper cites an incidence of $0.1 \%$ for device erosion with the
Amplatzer septal occluder in the USA. It is thought that an oversized device in a defect with a deficient aortic or superior rim may predispose to such a complication and these patients therefore merit closer and longer term follow up. Wire fractures are another reported complication of devices including the Helex and CardioSEAL/StarFLEX but seem to be without significant sequelae.

\section{COMPARISON OF TRANSCATHETER WITH SURGICAL CLOSURE OF ATRIAL SEPTAL DEFECTS}

It is difficult to carry out a robust scientific comparison of the two techniques. Nevertheless crude comparisons are available in the literature.

In a multicentre study from 29 paediatric cardiology centres, a comparison was made between transcatheter closure using the Amplatzer septal occluder in 442 patients and surgical closure in 154 patients. ${ }^{15}$ Although this was a non-randomised study, it gave a useful insight into the efficacy and complications associated with both procedures. Safety was defined as the absence of death or major complications. There were no deaths in either the device or surgical groups and the early success rates were not statistically different between the groups. Complications, classified as major or minor, occurred in 32/442 (7.2\%) of patients treated by transcatheter device closure and in 37/154 (24\%) treated surgically. The incidence of major complications was $7 / 442(1.6 \%)$ in the device treated group compared to $8 / 154(5.2 \%)$ in the surgically treated group $(\mathrm{p}=0.03)$. For the device group, device embolisation requiring surgical removal was the most common major complication, occurring in four patients. Cardiac arrhythmias requiring major treatment occurred in two patients. In the surgical group major complications included pulmonary oedema and large pericardial effusions requiring pericardiocentesis in two patients and prolonged intensive care unit stay in one patient; large pericardial effusion with tamponade requiring pericardiocentesis or catheter drainage in three patients; repeat surgery because of a large amount of drainage from the chest tube in two patients; and surgical wound complications requiring sternal wire removal and other treatments in two patients. Minor complications occurred in $27 / 442(6.1 \%)$ of the device treated group and in $29 / 154$ $(18.8 \%)$ of the surgically treated group $(p<0.001)$. Cardiac arrhythmia was the most common minor complication in both groups.

Generally these complications have not been highlighted in previous reports. Overall the literature supports the notion that treatment of secundum ASDs, whether by the traditional surgical method or by the newer transcatheter method, is associated with complications, but the trend is towards lower complications using the transcatheter method.

\section{CONCLUSIONS}

While it would be nice to state that with increasing experience, complications will be reduced to a minimum, the reality is otherwise. The increasing experience with transcatheter techniques has resulted in interventional cardiologists increasingly attempting to treat patients with ASDs of more complex morphology. In this subset of patients with complex defects the risk of complications may well be higher than in the majority of other patients with less challenging defects. Realistically, once an equilibrium of expertise and patient selection has been achieved, complications could be reduced but not completely abolished.

\section{Authors' affiliations}

M S Spence, S A Qureshi, Department of Congenital Heart Disease, Guy's \& St Thomas' Hospital, London, UK 


\section{REFERENCES}

1 Emmanoulides GC, Allen HD, Riemenschneider TA, et al. Heart disease in infants, children and adolescents, including the fetus and young adults. Baltimore: Williams and Wilkins, 1995:60-9.

2 Dickinson DF, Arnold R, Wilkinson JL. Congenital heart disease among 160 480 liveborn children in Liverpool 1960 to 1969. Implications for surgical treatment. Br Heart J 1981;46:55-62.

3 King TD, Thompson SL, Steiner C, et al. Secundum atrial septal defect. Nonoperative closure during cardiac catheterization. JAMA 1976;235:2506-9.

4 Murphy JG, Gersh BJ, McGoon MD, et al. Long-term outcome after surgical repair of isolated atrial septal defect. Follow-up at 27 to 32 years. N Engl J Med 1990;323:1645-50

5 Konstantinides S, Geibel A, Olschewski M, et al. A comparison of surgical and medical therapy for atrial septal defect in adults. N Engl J Med 1995:333:469-73.

6 Roos-Hesselink JW, Meijboom FJ, Spitaels SE, et al. Excellent survival and low incidence of arrhythmias, stroke and heart failure long-term after surgical atrial septal defect closure at young age. A prospective follow-up study of $21-$ 33 years. Eur Heart J 2003;24:190-7.

7 Baskett RJ, Tancock E, Ross DB. The gold standard for atrial septal defect closure: current surgical results, with an emphasis on morbidity. Pediatr Cardiol 2003;24:444-7.

8 Hopkins RA, Bert AA, Buchholz B, et al. Surgical patch closure of atrial septal defects. Ann Thorac Surg 2004;77:2144-9.
9 Shaw JA, Dewire E, Nugent A, et al. Use of suture-mediated vascular closure devices for the management of femoral vein access after transcatheter procedures. Catheter Cardiovasc Interv 2004;63:439-43.

10 Chessa M, Carminati M, Butera G, et al. Early and late complications associated with transcatheter occlusion of secundum atrial septal defect. J Am Coll Cardiol 2002;39:1061-5.

11 Fischer G, Stieh J, Uebing A, et al. Experience with transcatheter closure of secundum atrial septal defects using the Amplatzer septal occluder: a single centre study in 236 consecutive patients. Heart 2003:89:199-204.

12 Chan KC, Godman MJ, Walsh K, et al. Transcatheter closure of atrial septal defect and interatrial communications with a new self expanding nitinol double disc device (Amplatzer septal occluder): multicentre UK experience. Heart 1999:82:300-6.

13 Krumsdorf U, Ostermayer S, Billinger K, et al. Incidence and clinical course of thrombus formation on atrial septal defect and patient foramen ovale closure devices in 1,000 consecutive patients. J Am Coll Cardiol 2004:43:302-9.

14 Amin Z, Hijazi ZM, Bass JL, et al. Erosion of Amplatzer septal occluder device after closure of secundum atrial septal defects: review of registry of complications and recommendations to minimize future risk. Catheter Cardiovasc Interv 2004:63:496-502.

15 Du ZD, Hijazi ZM, Kleinman CS, et al. Amplatzer Investigators. Comparison between transcatheter and surgical closure of secundum atrial septal defect in children and adults: results of a multicenter nonrandomized trial. J Am Coll Cardiol 2002;39:1836-44.

\section{IMAGES IN CARDIOLOGY}

\section{Asymptomatic inferior vena cava anomaly in an adult: three dimensional multislice CT image}

A

52 year old male patient presented with an acute inferior myocardial infarction. His past medical history was unremarkable, and he was well up to 12 hours before presentation. He initially made an uneventful recovery during the first day, but on the second day postinfarction he developed symptomatic bradycardia necessitating temporary cardiac pacing, which was inserted via the right femoral vein under fluoroscopy. The pacing electrode catheter reached the right ventricular apex with an abnormal course (left panel), which forced us to consider an inferior vena cava (IVC) anomaly. On the seventh day of admission, three dimensional reconstructed multislice computed tomography of the whole thorax and abdomen delineated the complete absence of the right IVC trunk. The postrenal part of the IVC was present and transposed to the left side of the vertebral column, juxtaposing with the abdominal aorta (right panel). The other segments of the IVC-that is, the hepatic, prerenal, and renal parts-were also missing. The left renal vein drained directly into the left paravertebrally located IVC; by contrast, the right renal vein drained into the right azygos vein. Because of the absence of the hepatic segment of the IVC, all hepatic veins entered the inferior part of the right atrium. The enlarged right azygos vein joined the right superior vena cava at the right paratracheal space. All venous blood flow except from the hepatic veins entered the heart through the azygos and superior vena cava. This anatomical

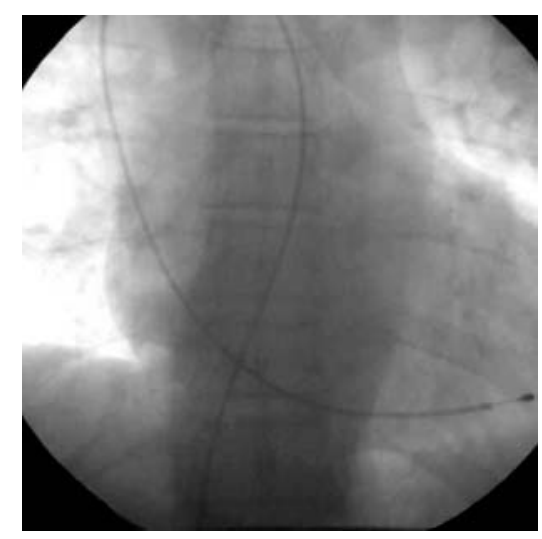

variant associated with renal vein drainage anomalies was consistent with IVC interruption and azygos continuation.

We describe an unusual case of an adult patient with IVC interruption and azygos continuation, in whom any symptoms or signs of complications of this anomaly were absent. This disorder has been described mainly as an incidental finding associated with a number of congenital malformations, especially with polysplenia syndrome. This anomaly is encountered in at least $65 \%$ of these patients. The incidence of congenital anomalies of the IVC is, however,

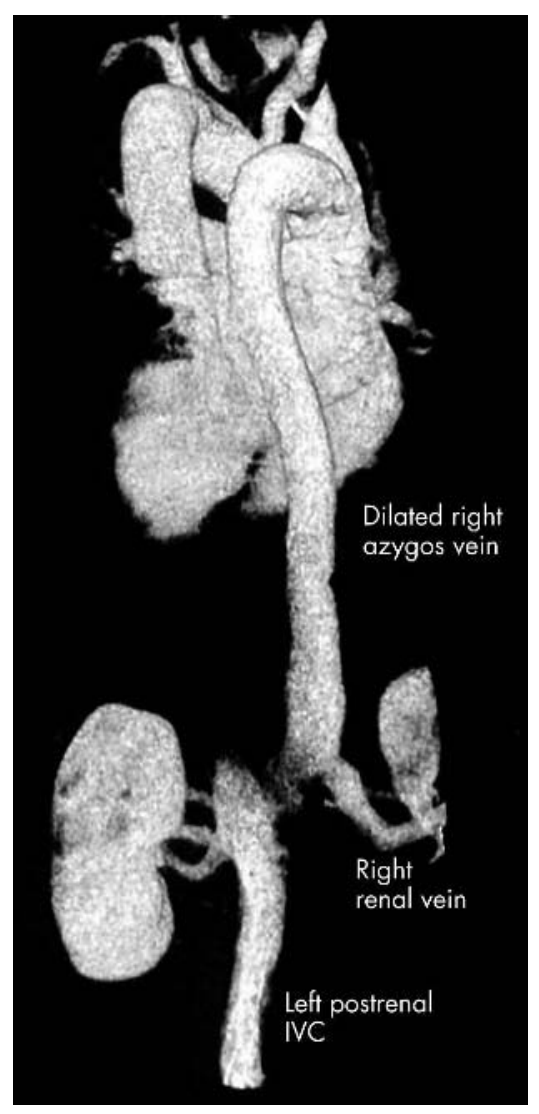
less than $1 \%$ in individuals who have no other congenital malformation.

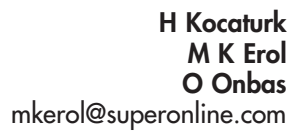

H Kocaturk

M K Erol

mkerol@superonline.com 